Pacific Journal of Mathematics

EXTENDING FUNCTIONS FROM PRODUCTS WITH A
METRIC FACTOR AND ABSOLUTES 


\title{
EXTENDING FUNCTIONS FROM PRODUCTS WITH A METRIC FACTOR AND ABSOLUTES
}

\author{
TEOdor C. PrZymusiński
}

\begin{abstract}
Extendability of continuous functions from products with a metric or a paracompact $p$-space factor is studied. We introduce and investigate completions $m X$ and $p X$ of a completely regular space $X$ defined as "largest" spaces $Y$ containing $X$ as a dense subspace such that every continuous real-valued function extends continuously from $X \times Z$ over $Y \times Z$ where $Z$ is a metric or a paracompact $p$-space, respectively. We study the relationship between $m X$ (resp. $p X)$ and the Hewitt realcompactification $v X$ (resp. the Dieudonné completion $\mu X$ ) of $X$. We show that for normal and countably paracompact spaces $m X=v X$ and $p X=\mu X$, but neither normality nor countable paracompactness alone suffices. The relationship between completions $m X$ and $p X$ and the absolute $E X$ of $X$ is discussed.
\end{abstract}

1. Introduction. All spaces are completely regular and all functions and mappings are continuous. Symbols $\boldsymbol{F}, \boldsymbol{M}, \boldsymbol{C}$ and $\boldsymbol{P}$ denote classes of finite spaces, metrizable spaces, compact spaces and paracompact $p$-spaces, respectively. We recall that $X$ is a paracompact $p$-space if it is a closed subspace of a product space $M \times C$, where $M$ is metrizable and $C$ is compact or-equivalently-if $X$ is an inverse image of a metrizable space under a perfect mapping. For all undefined notions the reader is referred to [3].

Let $X$ be a subspace of a space $Y$ and let $\tau$ be a cardinal number. We recall the definition of $P^{\tau}$-embedding of $X$ in $Y$. Our definition is equivalent to the original definition of this notion involving the extendability of continuous pseudometrics [see [10] for the proof and for more information].

If $\tau$ is infinite, then $X$ is $P^{-}$-embedded in $Y$ if every mapping $f: X \rightarrow B$ of $X$ into a Banach space $B$ of weight $\tau$ can be continuously extended over $Y$. If $\tau$ is finite, then $X$ is $P^{\tau}$-embedded in $Y$ if $X$ is $C^{*}$-embedded in $Y$. Moreover, $X$ is $P$-embedded in $Y$ if $X$ is $P^{-}$-

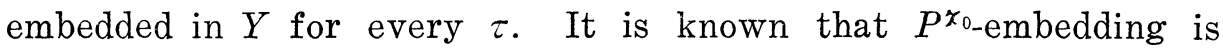
equivalent to $C$-embedding [4]. The following theorem gives a product-theoretic characterization of $P^{\tau}$-embedding. (X $\subset_{c^{*}} Y$ means that $X$ is $C^{*}$-embedded in $Y$, etc.)

Theorem 0 ([8], [10]). For a subspace $X$ of $Y$ and a cardinal number $\tau$ the following are equivalent:

(i ) $X \subset_{p^{*}} Y$; 
(ii) $X \times C \subset_{C^{*}} Y \times C$, for every $C \in C$ of weight $\tau$;

(iii) there exists a $C_{0} \in C$ of weight $\tau$ such that $X \times C_{0} \subset_{C^{*}} Y \times C_{0}$;

(iv) $X \times D^{\tau} \subset_{c^{*}} Y \times D^{\tau}$, where $D$ is the discrete two-point space.

Corollary 0 ([8], [10]). For a subspace $X$ of $Y$ the following are equivalent:

(i) $X \subset_{P} Y$;

(ii) $X \times C \subset_{C^{*}} Y \times C$, for every $C \in C$;

(iii) there exists a $C_{0} \in C$ of weight $\tau=|X|$ such that $X \times C_{0} \subset_{C^{*}} Y \times C_{0}$;

(iv) $X \times D^{\tau} \subset_{c^{*}} Y \times D^{\tau}$, where $\tau=|X|$.

The above stated results suggest the following definitions. By $Z$ we denote a nonempty class of spaces.

Definition 1. Let $X$ be a subspace of $Y$. We say that $X$ is

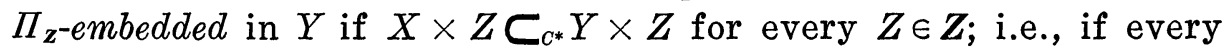
mapping $f: X \times Z \rightarrow I$ can be continuously extended over $Y \times Z$, for $Z \in Z$.

Definition 2. We say that a space $X$ is $\Pi_{z}$-complete if there is no space $Y$ containing $X$ as a proper, dense and $\Pi_{z}$-embedded subspace, i.e., if $X$ is closed in every space containing it as a $\Pi_{Z^{-}}$ embedded subspace.

Definition 3. We say that a space $Y$ is a $\Pi_{z^{-} \text {completion of } X}$ if $Y$ is a $\Pi_{Z^{-}}$-complete space containing $X$ as a dense $\Pi_{z}$-embedded subspace.

The following fact is easy to prove.

BASIC FACT. Every space $X$ has a uniquely determined $\Pi_{Z^{-}}$ completion, denoted by $\pi_{z} X$, and $\pi_{z} X=\left\{y \in \beta X: X \subset_{\pi_{Z}} X \cup\{y\}\right\}=$ $\cap\left\{Y: X \subset Y \subset \beta X\right.$ and $Y$ is $\Pi_{Z^{-}}$-complete).

It is the aim of this paper to characterize and investigate $\Pi_{z}$-embedding, $\Pi_{z}$-complete spaces and $\Pi_{z}$-completions $\pi_{z} X$ for the classes $\boldsymbol{M}$ and $\boldsymbol{P}$ of metric spaces and paracompact $p$-spaces, respectively. Let us put

$$
m X=\pi_{M} X \text { and } p X=\pi_{P} X .
$$


The table below illustrates the introduced concepts.

\begin{tabular}{|c|c|c|c|}
\hline$Z$ & $\Pi_{\boldsymbol{z} \text {-embedding }}$ & $\Pi_{\boldsymbol{Z} \text {-complete spaces }}$ & $\Pi_{z \text {-completion }} \pi_{z} X$ \\
\hline $\boldsymbol{F}$ & $C^{*}$-embedding & compact spaces & $\begin{array}{c}\beta X \\
\text { Cech-Stone } \\
\text { compactification }\end{array}$ \\
\hline $\boldsymbol{M} \cap \boldsymbol{C}$ & $C$-embedding & realcompact spaces & $\begin{array}{c}v X \\
\text { Hewitt real- } \\
\text { compactification }\end{array}$ \\
\hline$C$ & $P$-embedding & $\begin{array}{l}\text { Dieudonné-complete } \\
\text { spaces }\end{array}$ & $\begin{array}{c}\mu X \\
\text { Dieudonné completion }\end{array}$ \\
\hline$M$ & $?$ & $?$ & $?$ \\
\hline $\boldsymbol{P}$ & $?$ & $?$ & $?$ \\
\hline
\end{tabular}

Clearly, the following inclusions hold for any space $X$ :

\begin{tabular}{|c|c|c|}
\hline $\begin{array}{c}\pi_{F} X \\
U \\
\pi_{M} \cap C^{X} \\
\cup \quad \cup \\
\pi_{C} \quad \pi_{M^{X}} \\
\cup \quad U \\
\pi_{P}{ }^{X} \\
U \\
X\end{array}$ & $\begin{array}{c}\text { or } \\
\text { equivalently: }\end{array}$ & $\begin{array}{c}\beta X \\
\dot{U} \\
v X \\
U \cup \\
\mu X \quad m X \\
\cup U \\
p X \\
U \\
X\end{array}$ \\
\hline
\end{tabular}

It follows from well-known facts and the results proved in this paper that if measurable cardinals exist then all inclusions in the above diagram are in general proper and no other inclusions are generally valid. On the other hand, if the nonexistence of measurable cardinals is assumed, then the above diagram can be simplified as follows:

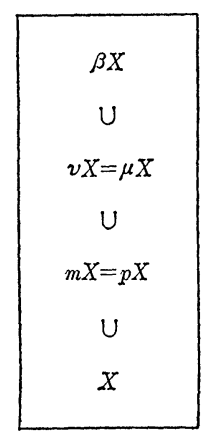

REMARK 1. It is pointless to investigate $\Pi_{z}$-completions for too broad classes of spaces. For example, if the class $\boldsymbol{Z}$ contains all spaces with one non-isolated point (in particular, if it contains all 
paracompact spaces), then $\pi_{z} X=X$ for every $X$ (cf. [7]; Theorem $5.2)$.

This paper consists of four sections. In $\S 2$ we present charac-

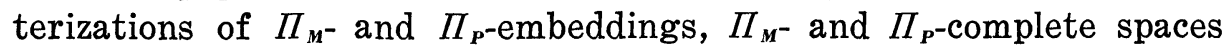

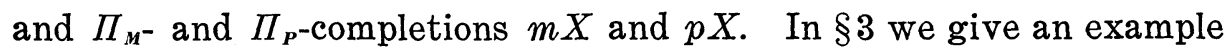
of a normal space $X$ such that $m X \neq v X$ and $p X \neq \mu X$. Section 4 is devoted to a discussion of the relationship existing between the above introduced concepts and absolutes of topological spaces. Several problems are raised.

2. Characterization theorems. Theorems 1 and 2 below give characterizations of $\Pi_{M^{-}}$and $\Pi_{P}$-embeddings (for dense subsets $X$ of $Y$ ). By $J(\tau)$ we denote the hedgehog with $\tau$ spikes (see [3], Example 4.1.5). A set $A$ is regularly open (regularly closed) if $A=\operatorname{Int} \bar{A}$ $(A=\overline{\operatorname{Int} A})$.

Theorem 1. For a dense subspace $X$ of $Y$ the following are equivalent:

(i) $X \subset_{\Pi_{M}} Y$;

(ii) $X \times Z \subset_{c^{*}} Y \times Z$, for every first countable $Z$;

(iii) $X \times J(\tau) \subset_{c^{*}} Y \times J(\tau)$, where $\tau=|X|$;

(iv) $X \subset_{C^{*}} Y$ and every regularly open increasing cover $\left\{U_{n}\right\}_{n<\omega}$ of $X$ can be extended over $Y$.

Proof. Implications (ii) $\Rightarrow$ (i) and (i) $\Rightarrow$ (iii) are obvious.

(iii) $\Rightarrow$ (iv). Let $\left\{U_{n}\right\}_{n<\omega}$ be an increasing regularly open cover of $X$ and for every $n$ let $F_{n}$ denote a closed set in $X$ such that $U_{n}=$ Int $F_{n}$. Since $X$ is completely regular there exist families $W_{n}=$ $\left\{W_{n, \alpha}\right\}_{\alpha<\tau}$ and $\boldsymbol{F}_{n}=\left\{F_{n, \alpha}\right\}_{\alpha<\tau}$ of cozero and zero sets, respectively, such that $F_{n} \subset F_{n, \alpha} \subset W_{n, \alpha}$ and $\bigcap_{\alpha<\tau} W_{n, \alpha}=F_{n}$. (Notice, that if the sets $F_{n}$ are zero sets, then we can require that the families $W_{n}$ and $\boldsymbol{F}_{n}$ be countable.) For every $n<\omega$ and $\alpha<\tau$ let $f_{n, \alpha}: X \rightarrow I$ be such that $f_{n, \alpha} \mid F_{n, \alpha} \equiv 0$ and $f_{n, \alpha} \mid\left(X \backslash W_{n, \alpha}\right) \equiv 1$. Represent $J(\tau)$ as the set $\{(t, \alpha): t \in I, \alpha<\tau\}$ with points $\{(0, \alpha): \alpha<\tau\}$ identified to a point $\theta$ and define a mapping $f: X \times J(\tau) \rightarrow I$ as follows. If $t=0$ then we put $f(x,(t, \alpha))=0$. If $t \in(0,1]$, then we can find an integer $n=1,2, \cdots$ such that $t \in[(1 / n+1),(1 / n)]$. There exists a unique $s \in[0,1]$ such that $t=s(1 /(n+1))+(1-s)(1 / n)$. Define for each $x \in X$ and $\alpha<\tau, f(x,(t, \alpha))=s . f_{n+1, \alpha}(x)+(1-s) \cdot f_{n, \alpha}(x)$. Note that if $t=1 / n$ for some integer $n$, the two possible values for $f(x,(t, \alpha))$ given by the above formula agree. Thus $f$ is well defined and is obviously continuous except perhaps at points of the form $(x, \theta)$. We now verify the continuity of $f$ at such points. Let $x \in X$. 
There exists an $n$ such that $x \in U_{n}$. Therefore, $x \in U_{n} \subset U_{k} \subset F_{k} \subset$ $F_{k, \alpha}$ for $k \geqq n$ and $\alpha<\tau$ and $f \mid U_{n} \times B_{n} \equiv 0$, where $B_{n}=\{(t, \alpha) \in$ $J(\tau): t<1 / n\}$.

By (iii) there exists a continuous extension $\tilde{f}: Y \times J(\tau) \rightarrow I$. To prove (iv) it is enough to check that $\bigcap_{n<\omega}{\overline{X \backslash U_{n}^{Y}}}_{n}=\varnothing$. Let $y_{0} \in Y$. Then $\widetilde{f}\left(y_{0}, 0\right)=0$ and there exists a neighborhood $W$ of $y_{0}$ in $Y$ and $n \geqq 1$ such that $\widetilde{f}\left(W \times B_{n-1}\right) \subset[0,1)$. We shall show that $W \cap X \subset U_{n}$. Suppose otherwise. Then $(W \cap X) \backslash U_{n} \neq \varnothing$ and thus $(W \cap X) \backslash F_{n} \neq \varnothing$. Choose $x_{0} \in(W \cap X) \backslash F_{n}$ and $\alpha<\tau$ such that $x_{0} \in X \backslash W_{n, \alpha}$. Then $f\left(x_{0},((1 / n), \alpha)\right)=f_{n, \alpha}\left(x_{0}\right)=1$, but $x_{0} \in W$ and $(1 / n, \alpha) \in B_{n-1}$. Contradiction.

(iv) $\Rightarrow$ (ii). Let $Z$ be a arbitrary first countable space and let $f: X \times Z \rightarrow I$. For every $y \in Y$ and $z \in Z$ put $\widetilde{f}(y, z)=\widetilde{f}_{z}(y)$, where $\widetilde{f}_{z}$ is the continuous extension over $Y$ of the function $f_{z}: X \rightarrow I$ defined by $f_{z}(x)=f(x, z)$. We shall show that the mapping $\tilde{f}: Y \times Z \rightarrow I$ is continuous. Let $y_{0} \in Y, z_{0} \in Z, \varepsilon>0$ and $\widetilde{f}\left(y_{0}, z_{0}\right)=s_{0}$. There exists a neighborhood $U$ of the point $y_{0}$ in $Y$ such that $\tilde{f}\left(U \times\left\{z_{0}\right\}\right) \subset$ $\left(s_{0}-(\varepsilon / 2), s_{0}+(\varepsilon / 2)\right)$. Let $K$ be a zero subeset of $Y$ such that $Y \backslash U \subset$ Int $K$ and $y_{0} \notin K$ and let $A$ be a dense subset of $Z$. For every $a \in A$ put

$$
K_{a}=\left\{x \in X:\left|f(x, a)-s_{0}\right| \leqq \frac{\varepsilon}{2}\right\}
$$

and for every $n<\omega$ define

$$
F_{n}=(K \cap X) \cup \cap\left\{K_{a}: a \in A \cap B_{n}\right\},
$$

where $\left\{B_{n}\right\}_{n<\omega}$ is a decreasing neighborhood base at $z_{0}$ in $Z$. The sets $F_{n}$ are closed in $X$ and nondecreasing. (Notice, that if $A$ is countable, then the sets $F_{n}$ are zero subsets of $X$.) The sets $U_{n}=$ Int $F_{n}$ are regularly open and nondecreasing. We shall show first that $X=\bigcup_{n<\omega} U_{n}$. If $x \in \operatorname{Int} K$, then $x \in U_{n}$ for every $n$. Otherwise, $x \in U$ and there exists a neighborhood $U_{x}$ of $x$ and $n<\omega$ such that

$$
f\left(U_{x} \times B_{n}\right) \subset\left(s_{0}-\varepsilon, s_{0}+\varepsilon\right) .
$$

Then $U_{x} \subset K_{a}$, for all $a \in A \cap B_{n}$ and thus $U_{x} \subset F_{n}$.

By (iv) there exist open sets $\widetilde{U}_{n}$ in $Y$ such that $\bigcup_{n<\omega} \widetilde{U}_{n}=Y$ and $\widetilde{U}_{n} \cap X=U_{n}$. Let $n$ be such that $y_{0} \in \widetilde{U}_{n}$. Since $y_{0} \notin K$ there exists a neighborhood $V$ of $y_{0}$ such that $V \cap X \subset U_{n} \backslash K$. By the continuity of $f$ we have

$$
f\left((V \cap X) \times B_{n}\right) \subset\left[s_{0}-\frac{\varepsilon}{2}, s_{0}+\frac{\varepsilon}{2}\right],
$$


and therefore by the continuity of functions $\widetilde{f}_{z}$ for $z \in Z$ and the density of $V \cap X$ in $V$ we get

$$
\tilde{f}\left(V \times B_{n}\right) \subset\left[s_{0}-\frac{\varepsilon}{2}, s_{0}+\frac{\varepsilon}{2}\right] \subset\left(s_{0}-\varepsilon, s_{0}+\varepsilon\right) .
$$

The following variant of Theorem 1 will be used in $\S 3$.

TheOREM 1*. For a dense subspace $X$ of $Y$ the following are equivalent:

(i) $X \times M \subset_{C^{*}} Y \times M$, for every separable $M \in M$;

(ii) $X \times Z \subset_{0^{*}} Y \times Z$, for every separable first countable space $Z$;

(iii) there exists a non-locally compact metric space $M_{0}$ such that $X \times M_{0} \subset_{C^{*}} Y \times M_{0}$;

(iv) $X \subset_{c^{*}} Y$ and every increasing open cover $\left\{U_{n}\right\}_{n<\omega}$ of $X$, such that $U_{n}=\operatorname{Int} F_{n}$ for some zero sets $F_{n}$, can be extended over $Y$.

Proof. Implications (ii) $\Rightarrow$ (i) and (i) $\Rightarrow$ (iii) are obvious. The proof of implications (iii) $\Rightarrow$ (iv) and (iv) $\Rightarrow$ (ii) is analogous to the proof of the corresponding implications in Theorem 1 (see the remarks in parentheses). One should only notice that every non-locally compact metric space contains as a closed subspace the subspace $J^{*}(\omega)=$ $\{(t, \alpha) \in J(\omega): t=0$ or $t=(1 / n)$ for some $n=1,2, \cdots\}$ of the hedgehog $J(\omega)$ and use the fact that for any space $T$, a closed subspace $F$ of a metric space $M$ and any mapping $h: F \times T \rightarrow I$ there exists a continuous extension $\tilde{h}: M \times T \rightarrow I$ [13].

THEOREM 2. For a dense subspace $X$ of $Y$ the following are equivalent:

(i) $X \subset_{\Pi P} Y$;

(ii) $X \times Z \subset_{C^{*}} Y \times Z$, for every space $Z$ of point-countable type $e^{1}$;

(iii) $X \times J(\tau) \times D^{\tau} \subset_{c^{*}} Y \times J(\tau) \times D^{\tau}$, where $\tau=|X|$;

(iv) $X \subset_{\Pi_{M}} Y$ and $X \subset_{\Pi_{C}} Y$.

Proof. The implications (ii) $\Rightarrow$ (i) and (i) $\Rightarrow$ (iii) are obvious. The implication (iii) $\Rightarrow$ (iv) follows from Theorems 0 and 1 .

(iv) $\Rightarrow$ (ii) Let $Z$ be a space of point-countable type and $f: X \times$ $Z \rightarrow I$. As in the proof of Theorem 1 we define $\widetilde{f}: Y \times Z \rightarrow I$ by putting $\widetilde{f}(y, z)=\widetilde{f}_{z}(y)$. We have to show that $\widetilde{f}$ is continuous. Let $y_{0} \in Y, z_{0} \in Z, \varepsilon>0$ and $\widetilde{f}\left(y_{0}, z_{0}\right)=s_{0}$. Let $C$ be a compact set of countable character in $Z$ containing $z_{0}$. By (iv) $\tilde{f} \mid Y \times C$ is continuous. Let $G=\left\{(y, z) \in Y \times C: \tilde{f}(y, z) \in\left(s_{0}-(\varepsilon / 2), s_{0}+(\varepsilon / 2)\right\}\right.$. The set $G$ is open in $Y \times C$ and contains $\left(y_{0}, z_{0}\right)$. Let us put $L=\left\{z \in C: \widetilde{f}\left(y_{0}, z\right)=s_{0}\right\}$.

${ }_{1}$ A space $Z$ is of point-countable type if for every $z_{0} \in Z$ there exists a compact $F \ni z_{0}$ of countable character in $Z$. 
The set $L$ is a zero set in $C$ and thus $L$ is of countable character in $Z$ and $z_{0} \in L$. Moreover, $\left\{y_{0}\right\} \times L \subset G$ and therefore there exists a neighborhood $U$ of $y_{0}$ in $Y$ such that $U \times L \subset G$. Let $K$ be a closed set in $Y$ such that $Y \backslash U \subset \operatorname{Int} K$ and $y_{0} \notin K$ and let $\left\{B_{n}\right\}_{n<\omega}$ be a decreasing base of neighborhoods of $L$ in $Z$. Since $f$ is continuous and $L$ is compact, for every $x \in U \cap X$ there exists a neighborhood $U_{x}$ and $n$ such that $f\left(U_{x} \times B_{n}\right) \subset\left(s_{0}-(\varepsilon / 2), s_{0}+(\varepsilon / 2)\right)$. Put $H_{n}=$ $\left\{x \in X: f\left(\{x\} \times B_{n}\right)\right) \subset\left[s_{0}-(\varepsilon / 2), s_{0}+(\varepsilon / 2\}\right]$. Of course, the sets $H_{n}$ are closed. Define

$$
U_{n}=\operatorname{Int}\left(H_{n} \cup(K \cap X)\right) .
$$

The sets $U_{n}$ are regularly open, nondecreasing and cover $X$, hence by (iv) and Theorem 1 there exists an $n$ and an open set $V \ni y_{0}$ in $Y$ such that $V \cap X \subset U_{n}$ and $V \cap K=\varnothing$. Then $f\left((V \cap X) \times B_{n}\right) \subset$ $\left[s_{0}-(\varepsilon / 2), s_{0}+(\varepsilon / 2)\right]$ and consequently $\tilde{f}\left(V \times B_{n}\right) \subset\left[s_{0}-(\varepsilon / 2), s_{0}+(\varepsilon / 2)\right] \subset$ $\left(s_{0}-\varepsilon, s_{0}+\varepsilon\right)$.

Corollary 1. For every $X$ we have $p X=m X \cap \mu X$.

Proof. $\quad p X=\pi_{P} X=\pi_{M} X \cap \pi_{C} X=m X \cap \mu X$.

COROLLARY 2. If there are no measurable cardinals, then $p X=$ $m X$ for every $X$.

CoROllary 3. For every $X$ the following are equivalent:

(i) $\mu(X \times M)=\mu X \times M$, for every $M \in M$;

(ii) $\mu(X \times P)=\mu X \times P$, for every $P \in P$;

(iii) $\mu X=p X$.

Proof. The implication (ii) $\Rightarrow$ (i) is obvious. If (i) holds, then $X \times M$ is $C^{*}$-embedded in $\mu X \times M$ for every $M \in M$ and therefore $\mu X \subset m X$ and $p X=\mu X \cap m X=\mu X$.

If (iii) holds, then $X \times P \times P^{\prime}$ is $C^{*}$-embedded in $\mu X \times P \times P^{\prime}$ for every $P, P^{\prime} \in P$ which implies that $X \times P$ is $P$-embedded in $\mu X \times P$.

The following three corollaries can be easily derived from Theorem 1.

CoRollary 4. A point $y \in \beta X$ belongs to $m X$ if and only if for every decreasing sequence $\left\{F_{n}\right\}_{n<\omega}$ of regularly closed subsets of $X$ with empty intersection $y \notin \bigcap_{n<\omega} \bar{F}_{n}^{\beta X}$.

Corollary 5. A space $X$ is $\Pi_{M^{-}}$complete if and only if for 
every $y \in \beta X \backslash X$ there exists a decreasing sequence $\left\{F_{n}\right\}_{n<\omega}$ of regularly closed subsets of $X$ such that $y \in \bigcap_{n<\omega} \bar{F}_{n}^{\beta X} \subset \beta X \backslash X$.

Corollary 6. A normal space $X$ is $\Pi_{M^{-}}$complete if and only if every closed ultrafilter in $X$, such that every decreasing sequence of its regularly closed elements has a nonempty intersection, converges to a point of $X$.

REMark 2. Since characterizations of $P$-embedding, Dieudonnécomplete spaces and Dieudonné completions are well known, Corollary 1 and Corollaries 4,5, and 6 yield immediately characterizations of $\Pi_{P}$-complete spaces and the $\Pi_{P}$-completion $p X$.

It is easy to verify that the assumption in Theorem 1 that the sequence $\left\{U_{n}\right\}_{n<\omega}$ is increasing is essential.

In [2] N. Dykes introduced the concept of c-realcompact spaces and c-realcompactification $u X$ of a space $X$. Later, these concepts were inverstigated by $K$. Hardy and R. Woods in [5] and [14], where new characterizations of $u X$ were obtained and the relationship between the $c$-realcompactification $u X$ and the absolute of $X$ was established. It follows from Corollary 4 and Lemma 1.1 from [5] that the concepts of $c$-realcompact spaces and $\Pi_{M}$-complete spaces are identical and that $u X=m X$ for every $X$. (We shall discuss the relationship between completions $m X$ and $p X$ and the absolute of $X$ in $\S 4)$. The following two results were known for $c$-realcompactification $u X$ (see [2] and [5]).

Corollary 7. Suppose that $X$ is normal and countably paracompact. Then:

$$
m X=v X \text { and } p X=\mu X .
$$

In particular, $X$ is $\Pi_{M^{-}}$complete iff $X$ is realcompact and $X$ is $\Pi_{P^{-}}$ complete iff $X$ is Dieudonné-complete.

Proof. Always $m X \subset v X$ and $\mu X \subset p X$. Let $y \in v X \subset \beta X$ and let $\left\{F_{n}\right\}_{n<\omega}$ be a decreasing sequence of regularly closed subsets of $X$ such that $\bigcap_{n<\omega} F_{n}=\varnothing$. There exists a sequence $\left\{K_{n}\right\}_{n<\omega}$ of zero subsets of $X$ such that $F_{n} \subset K_{n}$ and $\bigcap_{n<\omega} K_{n}=\varnothing$. Let $f_{n}: X \rightarrow I$ be functions such that $f_{n}^{-1}(0)=K_{n}$ and let $\widetilde{f}_{n}: \beta X \rightarrow I$ be continuous extensions. Then the function $\widetilde{f}=\sum_{n<\omega}\left(1 / 2^{n}\right) \widetilde{f}_{n}: \beta X \rightarrow I$ is continuous and $\tilde{f}^{-1}(0)=\bigcap_{n<\omega} \bar{K}_{n}^{\beta X} \subset \beta X \backslash X$. Therefore $\tilde{f}^{-1}(0) \cap \nu X=\varnothing$ and $y \notin \bigcap_{n<\omega} \bar{F}_{n}^{\beta . X} \subset \bigcap_{n<\omega} \bar{K}_{n}^{\beta, X}$, which in view of Corollary 4 shows that $y \in m X$.

Since $p X=\mu X \cap m X=\mu X \cap \cup X$ and $\cup X \supset \mu X$, we have $p X=\mu X$. 
CoROLlary 8. The following are equivalent:

(i) $X$ is psedocompact;

(ii) $m X=\beta X$;

(iii) $p X=\beta X$.

Proof. Implications (iii) $\Rightarrow$ (ii) $\Rightarrow$ (i) are obvious. If $X$ is pseudocompact, then clearly $\mu X=\beta X$ and since $p X=m X \cap \mu X$, it suffices to show that $m X=\beta X$, but every decreasing sequence of regularly closed subsets of a pseudocompact space is finite and thus $\beta X \subset m X$ by Corollary 4.

Let us finish this section with two problems.

Problem 1. Characterize closed $\Pi_{M}$-embedded ( $\Pi_{P}$-embedded) subspaces of a space $X$. Is it true that a closed subset of a space $X$ is $\Pi_{P}$-embedded iff it is $\Pi_{M}$-embedded and $\Pi_{C}$-embedded $(=P$ embedded) in $X$ ?

Problem 2. Investigate the $\Pi_{L}$-completion $\pi_{L} X$ of a space $X$ for the class $L$ of Lindelöf spaces.

3. An example. As yet no example was given of a space $X$ such that $m X \neq v X$ or $p X \neq \mu X$. In view of Corollary 7 , such a space cannot be normal and countably paracompact. It follows from the properties of the example in [6], the identity $u X=m X$ and Theorem 1.11 from [5] that there exists a countably paracompact space $X$ such that $\cup X=\mu X \neq m X=p X$. (Moreover, the space $X$ is locally compact and $v X$ is $\sigma$-compact.) Below we shall give an example of a normal space with analogous properties, thus showing that normality of $X$ is not sufficient in Corollary 7. Our example will be a modification of M. E. Rudin's example [12].

EXAMPLE 1. There exists a collectionwise normal space $X$ such that the space $v X=\mu X$ is paracompact and for every metric space $M$ we have:

(*) $\quad X \times M \subset_{c^{*}} \cup X \times M$ iff $M$ is locally compact.

In particular, $v X=\mu X \neq m X=p X$ and for every metric $M$ we have:

$$
\mu(X \times M)=\mu X \times M \text { iff } M \text { is locally compact . }
$$

REMARK 3. The existence of a (nonnormal) space satisfying (**) follows from results of $\mathrm{H}$. Ohta [9]. 
LEMMA 1. It suffices to construct a collectionwise normal space $X$ such that:

(a) the space $\cup X=\mu X$ is paracompact;

(b) there exists an increasing regularly open cover $\left\{U_{n}\right\}_{n<\omega}$ of $X$ which does not have an open locally finite refinement and such that the sets $\bar{U}_{n}$ are zero subsets of $X$.

Proof. Clearly $X \times M \subset_{C^{*}} \cup X \times M$ for every locally compact $M$ (see [1]). Conversely, suppose that $X \times M_{0} \subset_{C^{*}} \cup X \times M_{0}$ for some non-locally compact space $M_{0}$. From Theorem $1^{*}$ it follows that the open cover $\left\{U_{n}\right\}_{n<\omega}$ can be extended over $\cup X$ and since $v X$ is paracompact, it must have a locally finite open refinement. Contradiction.

By [12] there exists a collectionwise normal space $Y$ of nonmeasurable cardinality such that the space $v Y=\mu Y$ is paracompact and an increasing open cover $\left\{V_{n}\right\}_{n<\omega}$ of $Y$, which does not have a locally finite open refinement.

Let $Z$ be a closed subspace of the space $Y \times \omega$ (where $\omega$ bears the discrete topology) defined by $Z=\mathbf{U}_{n<\omega}\left(\bar{V}_{n} \times\{n\}\right)$ and let $W_{n}=$ $Z \cap\left(V_{n} \times\{1,2, \cdots, n\}\right)$. One easily sees (cf. [11]), that $Z$ is collectionwise normal, the space $v Z=\mu Z$ is paracompret, the sets $\bar{W}_{n}$ are zero subsets of $Z$ and the increasing open cover $\left\{W_{n}\right\}_{n<\omega}$ of $Z$ does not have a locally finite open refinement. Observe, that the sets $W_{n}$ need not be regularly open.

Now, let $X=Z \times I$, where points $(z, t) \in X$ are isolated if $t \neq 0$ and have a base of standard product neighborhoods if $t=0$ (cf. [11]). One easily checks that the space $X$ is collectionwise normal, the sets $U_{n}=W_{n} \times I$ form a regularly open covering of $X$ with no locally finite open refinement and the sets $\bar{U}_{n}$ are zero subsets of $X$. By Lemma 1 it suffices to show that the space $v X=\mu X$ is paracompact.

Let $T=\{(y, t) \in \cup Z \times I: y \in Z$ if $t \neq 0\}$ be considered with the topology in which points $(y, t) \in T$ are isolated if $t \neq 0$ and basic neighborhoods of a point $(y, 0) \in T$ are of the form $\pi^{-1}(U) \backslash K$, where $U$ is a neighborhood of $y$ in $v Z, \pi: T \rightarrow v Z$ is the projection and $K$ is a closed subset of $X=Z \times I$ contained in $Z \times(0,1]$. It is not difficult to verify that the space $T$ is paracompact and contains $X$ as a dense subspace. To show that $v X=\mu X=T$ it suffices to show that $X$ is $C$-embedded in $T$.

Let $f: X \rightarrow R$ and let $\tilde{f}: T \rightarrow R$ be an extension of $f$ defined by $\widetilde{f}(y, 0)=\widetilde{g}(y)$ for $y \in \cup Z$, where $\widetilde{g}: \cup Z \rightarrow R$ is the extension over $\cup Z$ of the function $g: Z \rightarrow R$ defined by $g(z)=f(z, 0)$. We have to show that $\tilde{f}$ is continuous. Let $y_{0} \in \cup Z, \varepsilon>0$ and $\tilde{f}\left(y_{0}, 0\right)=s_{0}$. The set 
$K_{1}=\left\{x \in X:\left|f(x)-s_{0}\right| \geqq \varepsilon\right\}$ is closed in $X$. Let

$$
K_{2}=\left\{y \in \cup Z:\left|\widetilde{f}(y, 0)-s_{0}\right| \leqq \frac{\varepsilon}{2}\right\} \times I .
$$

The set $K=K_{1} \cap K_{2}$ is closed in $X$ and contained in $Z \times(0,1]$. Let $W=\pi^{-1}\left(\left\{y \in \cup Z:\left|\widetilde{f}(y, 0)-s_{0}\right|<\varepsilon / 2\right\}\right) \backslash K$. The set $W$ is an open neighborhood of $\left(y_{0}, 0\right)$ in $T$ and $\widetilde{f}(W) \subset\left(s_{0}-\varepsilon, s_{0}+\varepsilon\right)$, which completes the proof.

4. Relationship with absolutes. For information about absolutes of topological spaces we recommend [15]. Here, we only recall that for every space $X$ there exists a uniquely determined extermally disconnected space $E X$ called the absolute of $X$ such that $E X$ can be mapped by a perfect irreducible mapping $k_{X}$ onto $X$.

If the space $Z$ is compact, then $E Z$ is the set of all ultrafilters in the Boolean algebra $R(Z)$ of all regularly closed subsets of $Z$ with the topology generated by the base $\{\lambda(F): F \in \boldsymbol{R}(Z)\}$, where $\lambda(F)=$ $\{p \in E Z: F \in p\}$.

The mapping $k_{Z}: E Z \rightarrow Z$ is defined by $k_{z}(p)=z$ iff $\{z\}=\cap p$. The sets $\lambda(F)$, for $F \in \boldsymbol{R}(Z)$, constitute all clopen subsets of the (compact) space $E Z$.

In general, $E X$ is the inverse image of $X$ under the mapping $k_{\beta X}: E(\beta X) \rightarrow \beta X$ and $k_{X}=k_{\beta_{X}} \mid E X$. The space $E X$ is dense in $E(\beta X)$. We put $\lambda^{*}(K)=\lambda\left(\bar{K}^{\beta X}\right) \cap E X$ for all $K \in \boldsymbol{R}(X)$.

It is well-known that $E(\beta X)=\beta(E X)$ for every space $X$ and that always $v(E X) \subset E(\nu X)$ and $\mu(E X) \subset E(\mu X)$. The following result has been proved by Hardy and Woods (we replace everywhere $u X$ by $m X)$. Here $k$ denotes the mapping $k_{\beta X}: E(\beta X) \rightarrow \beta X$.

THEOREM 3 [5], [14]. The following are equivalent

(i) $v(E X)=E(v X)$.

(ii) $v X=m X$.

More precisely, $m X$ is the largest subspace $T$ of $\beta X$ such that $k^{-1}(T) \subset v(E X)$.

We were unable to establish if the analogous fact holds for $\mu X$ and $p X$. However, the following two propositions are true. We denote by $s X$ the largest subspace $T$ of $\beta X$ such that $k^{-1}(T) \subset \mu(E X)$.

Proposition 1. A point $y \in \beta X$ belongs to $s X$ if and only if for every locally finite regularly closed cover $\left\{F_{s}\right\}_{s \in S}$ of $X$ there exist $s_{1}, \cdots, s_{n} \in S$ such that $y \in \operatorname{Int}_{\beta_{X}} \bigcup_{i=1}^{n} \bar{F}_{s_{i}}^{\beta X}$.

Proposition 2. Always $s X \subset p X$. 
Before proving Propositions 1 and 2 let us note that $s X=\mu X$ if and only if $\mu(E X)=E(\mu X)$ and thus if $\mu(E X)=E(\mu X)$, then $p X=\mu X$. Two natural problems arise:

Problem 3. Is always $s X=p X$ ?

Problem 4. Is $p X=\mu X$ equivalent to $\mu(E X)=E(\mu X)$ ? (Naturally, a positive answer to the first question answers positively the second.)

Proof of Proposition 1. Suppose that $y \in s X$ and let $\left\{F_{s}\right\}_{s \in S}$ be a locally finite regularly closed cover of $X$. Then the family $\left\{\lambda^{*}\left(F_{s}\right): s \in S\right\}$ is a locally finite clopen cover or $E X^{2}$. Since $k^{-1}(y) \subset$ $\mu(E X)$, there exist indices $s_{1}, \cdots, s_{n} \in S$ such that

$$
k^{-1}(y) \subset \bigcup_{i=1}^{n} \overline{\lambda^{*}\left(F_{s_{i}}\right)^{\beta(E X)}} \text {. }
$$

But $\beta(E X)=E(\beta X)$ and therefore $\left.\overline{\lambda^{*}\left(F_{s}\right.}\right)^{\beta(E X)}=\lambda\left(\bar{F}_{s}^{\beta(X)}\right)$ for all $s \in S$. Consequently, $k^{-1}(y) \subset \bigcup_{i=1}^{n} \lambda\left(\bar{F}_{s_{i}}^{\beta X}\right)$ and since $k$ is a closed mapping, there exists an open set $U \in y$ in $\beta X$ such that $k^{-1}(U) \subset \bigcup_{i=1}^{n} \lambda\left(\bar{F}_{s_{i}}^{\beta X}\right)$. Then $y \in U \subset \bigcup_{i=1}^{n} \bar{F}_{s_{i}}^{\beta X}$.

Conversely, suppose that $p \in k^{-1}(y)$ and let $\boldsymbol{U}=\left\{U_{s}\right\}_{s \in S}$ be a locally finite cozero cover of $E X$. Since $E X$ is extremally disconnected, we can assume that $U$ is pairwise disjoint and we have to show that there exists an $s \in S$ such that $p \in \bar{U}_{s}^{\beta(E X)}$. Since the sets $U_{s}$ are clopen, there exist regularly closed sets $F_{s}$ in $X$ such that $U_{s}=\lambda^{*}\left(F_{s}\right)$ and clearly $\bar{U}_{s}^{\beta(E X)}=\lambda\left(\bar{F}_{s}^{\beta X}\right)$. The family $\left\{F_{s}\right\}_{s \in S}$ is a regularly closed cover of $X$ and thus there exist $s_{1}, \cdots, s_{n} \in S$ such that $y \in$ $\operatorname{Int}_{\beta_{X}} \bigcup_{i=1}^{n} \bar{F}_{s_{i}}^{\beta X}$. Therefore, $\bigcup_{i=1}^{n} \bar{F}_{s_{i}}^{\beta X} \in p$ and since $p$ is an ultrafilter, there exists an $i$ such that $\bar{F}_{s_{i}}^{\beta X} \in p$ which means that $p \in \lambda\left(\bar{F}_{s_{i}}^{\beta X}\right)=$ $\bar{U}_{s_{i}}^{\beta(E X)}$.

Proof of Proposition 2. By Theorem 3, $s X \subset m X$ and since $p X=m X \cap \mu X$ it is enough to show that $s X \subset \mu X$. Let $y \in s X$ and let $\left\{U_{s}\right\}_{s \in S}$ be a locally finite cozero cover of $X$. We have to show that there exists an $s \in S$ such that $y \in \bar{U}_{s}^{\beta X}$. Let $\left\{V_{s}\right\}_{s \in S}$ be a covering of $X$ such that $V_{s} \subset \bar{V}_{s} \subset U_{s}$ for every $s \in S$. The family $\left\{\bar{V}_{s}\right\}_{s \in S}$ is a locally finite regularly closed cover of $X$ and thus there exist $s_{1}, \cdots, s_{n} \in S$ such that $y \in \bigcup_{i=1}^{n} \bar{V}_{s_{i}}^{\beta X}$. Therefore, there exists an $i$ such that $y \in \bar{V}_{s_{i}}^{\beta X} \subset \bar{U}_{s_{i}}^{\beta X}$.

Added in proof. Professor H. Ohta proved that a positive answer to Problems 3 and 4 above is equivalent to the non-existence of

${ }^{2}$ To show that this is a cover of $E X$, we use the local finiteness of $\left\{F_{s}\right\}_{s \in S}$. 
measurable cardinals. He also-independently-obtained some of the results in this paper. The interested should consult his paper: (1) The Hewitt real-compactification of products, Trans. AMS 263 (1981), 363-375; (2) Local compactness and Hewitt real-compactifications of products II, to appear; (3) Topological extension properties and projective covers, to appear; and also his $\mathrm{Ph}$. $\mathrm{D}$. Thesis at the University of Tsukuba, 1979.

For new results involving $\Pi_{z}$-embeddings, the reader is referred to the paper by A. Waśko, Extension of functions defined on product spaces, to appear in Fund. Math.

\section{REFERENCES}

1. W. W. Comfort, On the Hewitt realcompactification of a product space, Trans. Amer. Math. Soc., 131 (1968), 107-118.

2. N. Dykes, Generalizations of realcompact spaces, Pacific J. Math., 33 (1970), 571-581.

3. R. Engelking, General Topology, Polish Scientific Publishers, Warsaw. 1977.

4. T. E. Gantner, Extensions of uniformly continuous pseudometrics, Trans. Amer.

Math. Soc., 132 (1968), 147-157.

5. K. Hardy and R. G. Woods, On c-realcompact spaces and locally bounded normal functions, Pacific J. Math., 43 (1972), 647-656.

6. J. E. Mack and D. G. Johnson, The Dedekind completion of $C(X)$, Pacific J. Math., 20 (1967), 231-243.

7. W. G. McArthur, Hewitt realcompactifications of products, Canad. J. Math., 22 (1970), 645-656.

8. K. Morita and T. Hoshina, P-embedding and product spaces, Fund. Math., 93 (1976), $71-80$.

9. H. Ohta, Local compactness and Hewitt realcompactifications of products, Proc. Amer. Math. Soc., 69 (1978), 339-343.

10. T. C. Przymusinski, Collectionwise normality and extensions of continuous functions, Fund. Math., 98 (1978), 75-81.

11. - On locally finite coverings, Colloq. Math., 38 (1978), 187-192.

12. M. E. Rudin, A normal space $X$ for which $X \times I$ is not normal, Fund. Math., 73 (1971), 179-186.

13. M. Starbird, Extending maps from products, in: Studies in Topology, Academic Press, 1975, 559-565.

14. R. G. Woods, A Tychonoff almost real-compactification, Proc. Amer. Math. Soc., 43 (1974), 200-208.

15. - A survey of absolutes of topological spaces, in: Topological Structures II, Math. Centre Tracts, 116 (1979), 323-362.

Received May 21, 1980 and in revised form February 20, 1981.

Polish ACAdemy of Sciences,

00-950 WarsaW, SNIADECKICH 8,

POLAND

Current address: Math. Dept., Unlversity of Pittsburgh, PA 15260. 



\section{PACIFIC JOURNAL OF MATHEMATICS}

\section{EDITORS}

DONALD BABBITT (Managing Editor)

University of California

Los Angeles, CA 90024

Hugo Rossi

University of Utah

Salt Lake City, UT 84112

C. C. MOore and Arthur Agus

University of California

Berkeley, CA 94720
J. DugundJI

Department of Mathematics

University of Southern California

Los Angeles, CA 90007

R. FinN and J. MILGRAM

Stanford University

Stanford, CA 94305

\section{ASSOCIATE EDITORS}
R. ARENS
E. F, BECKENBACH
B. H. NeumanN
F. WOLF
K. YoSHIDA

\section{SUPPORTING INSTITUTIONS}

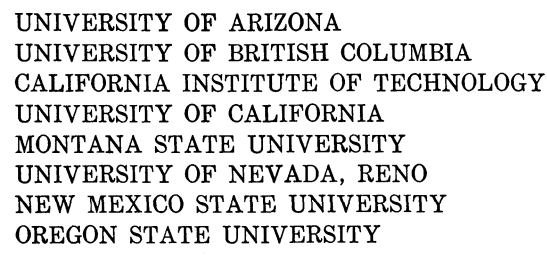

UNIVERSITY OF ARIZONA

UNIVERSITY OF BRITISH COLUMBIA

CALIFORNIA INSTITUTE OF TECHNOLOGY

UNIVERSITY OF CALIFORNIA

MONTANA STATE UNIVERSITY

UNIVERSITY OF NEVADA, RENO

NEW MEXICO STATE UNIVERSITY

OREGON STATE UNIVERSITY

\author{
UNIVERSITY OF OREGON \\ UNIVERSITY OF SOUTHERN CALIFORNIA \\ STANFORD UNIVERSITY \\ UNIVERSITY OF AAWAII \\ UNIVERSITY OF TOKYO \\ UNIVERSITY OF UTAH \\ WASHINGTON STATE UNIVERSITY \\ UNIVERSITY OF WASHINGTON
}

The Supporting Institutions listed above contribute to the cost of publication of this Journal, but they are not owners or publishers and have no responsibility for its content or policies,

Mathematical parers intended for publication in the Pacific Journal of Mathematics should be in typed form or offset-reproduced, (not dittoed), double spaced with large margins. Please do not use built up fractions in the text of the manuscript. However, you may use them in the displayed equations. Underline Greek letters in red, German in green, and script in blue. The first paragraph or two must be capable of being used separately as a synopsis of the entire paper. Please propose a heading for the odd unmbered pages of less than 35 characters. Manuscripts, in triplicate, may be sent to any one of the editors. Please classify according to the scheme of Math. Reviews, Index to Vol. 39. Supply name and address of author to whom proofs should be sent. All other communications should be addressed to the managing editor, or Elaine Barth, University of California, Los Angeles, California, 90024.

50 reprints to each author are provided free for each article, only if page charges have been substantially paid. Additional copies may be obtained at cost in multiples of 50 .

The Pacific Journal of Mathematics is issued monthly as of January 1966, Regular subscription rate: $\$ 114.00$ a year $(6$ Vol., 12 issues). Special rate: $\$ 57.00$ a year to individual members of supporting institution.

Subscriptions, orders for numbers issued in the last three calendar years, and changes of address shoud be sent to Pacific Journal of Mathematics, P.O. Box 969, Carmel Valley, CA 93924, U.S.A. Old back numbers obtainable from Kraus Periodicals Co., Route 100, Millwood, NY 10546.

PUBLISHED BY PACIFIC JOURNAL OF MATHEMATICS, A NON-PROFIT CORPORATION

Printed at Kokusai Bunken Insatsusha (International Academic Printing Co., Ltd.). 8-8, 3-chome, Takadanobaba, Shinjuku-ku, Tokyo 160, Japan.

Copyright (C) 1982 by Pacific Journal of Mathematics Manufactured and first issued in Japan 


\section{Pacific Journal of Mathematics}

\section{Vol. 101, No. 2 December, 1982}

Jean Bourgain, A Hausdorff-Young inequality for $B$-convex Banach

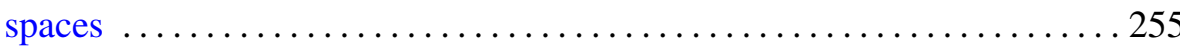

J. L. Brenner and Lorraine L. Foster, Exponential Diophantine

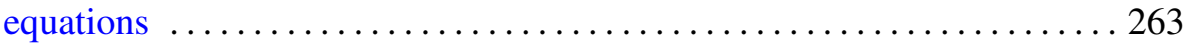

Henry H. Glover and William Duncan Homer, II, Fixed points on flag

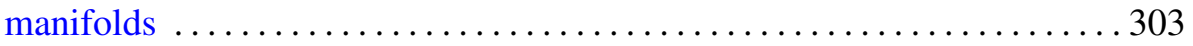

Lothar Hahn, A note on stochastic methods in connection with approximation theorems for positive linear operators $\ldots \ldots \ldots \ldots \ldots . \ldots 307$

James P. Henderson, Approximating cellular maps between low-dimensional polyhedra

V. K. Jain, Certain transformations of basic hypergeometric series and their

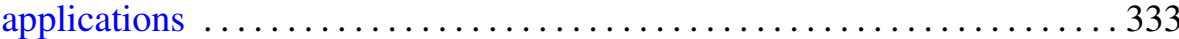

Charles David Keys, On the decomposition of reducible principal series

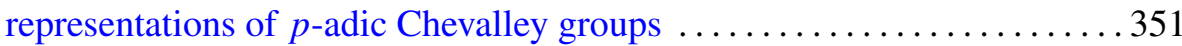

M. S. Klamkin and A. Meir, Ptolemy's inequality, chordal metric,

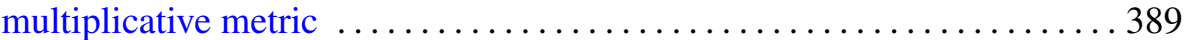

Robert F. Lax, Independence of normal Weierstrass points under

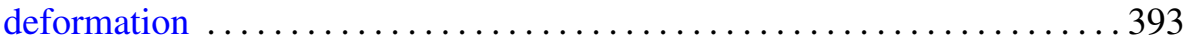

Leonid A. Luxemburg, On compactifications of metric spaces with

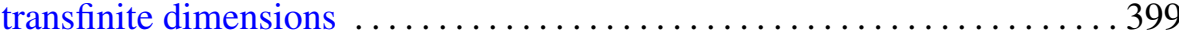

Carlton James Maxson, Martin Ross Pettet and Kirby C. Smith, On semisimple rings that are centralizer near-rings .....

Teodor C. Przymusiński, Extending functions from products with a metric factor and absolutes

Giorgio Talenti, A note on the Gauss curvature of harmonic and minimal surfaces

D. M. Terlinden, A spectral containment theorem analogous to the semigroup theory result $e^{t \sigma(A)} \subseteq \sigma\left(e^{t A}\right)$ 